\title{
Vibrotactile Letter Reading Using a Low-Resolution Tactor Array
}

\author{
Yasuyuki Yanagida ${ }^{1}$, Mitsuhiro Kakita ${ }^{1,2}$, Robert W. Lindeman ${ }^{3,1}$, \\ Yuichiro Kume ${ }^{4,1}$, and Nobuji Tetsutani ${ }^{1}$ \\ ${ }^{1}$ Media Information Science Laboratories \\ ATR \\ Kyoto, Japan \\ \{yanagida $\mid$ mitsu $\mid$ tetsutani\}@atr.jp \\ ${ }^{3}$ Department of Computer Science \\ George Washington University \\ Washington DC, USA \\ gogo@gwu.edu \\ ${ }^{2}$ School of Knowledge Science \\ Japan Advanced Institute of Science \& Technology \\ Ishikawa, Japan \\ ${ }^{4}$ Faculty of Engineering \\ Tokyo Polytechnic University \\ Kanagawa, Japan \\ kume@photo.t-kougei.ac.jp
}

\begin{abstract}
Vibrotactile displays have been studied for several decades in the context of sensory substitution. Recently, a number of vibrotactile displays have been developed to extend sensory modalities in virtual reality. Some of these target the whole body as the stimulation region, but existing systems are only designed for discrete stimulation points at specific parts of the body. However, since human tactile sensation has more resolution, a higher density might be required in tactor alignment in order to realize general-purpose vibrotactile displays. One problem with this approach is that it might result in an impractically high number of required tactors. Our current focus is to explore ways of simplifying the system while maintaining an acceptable level of expressive ability. As a first step, we chose a well-studied task: tactile letter reading. We examined the possibility of distinguishing alphanumeric letters by using only a 3-by3 array of vibrating motors on the back of a chair. The tactors are driven sequentially in the same sequence as if someone were tracing the letter on the chair's back. The results showed $87 \%$ successful letter recognition in some cases, which was close to the results in previous research with much larger arrays.
\end{abstract}

\section{Introduction}

The main target region of tactile displays in virtual reality has been the fingertips, usually used for object handling. Humans, however, perceive the surrounding environment by using the whole body, as well as the fingertips. Recently, researchers have been trying to expand the region of stimulation by tactile displays to the whole body.
Some of these are suit-type displays that cover the whole body, but existing systems only target discrete stimulation points, such as the shoulders, elbows, wrists, thighs, knees, and so on. These could be useful for some applications such as alert messages of simple collision against objects or walls in virtual environments, but they are not sufficient to convey rich information by vibrotactile sensation.

Another approach to body-oriented vibrotactile displays is to place many tactors at a certain region of the body at higher spatial density. This approach has historically been attractive, and it is pervasive in sensory substitution research. This approach made it possible to display rich information, but the target region was limited to one region of the body because it was technically difficult to place a large number of tactors over the whole body.

Our interest lies in finding a way to balance these two approaches. We want to reduce the number of tactors as much as possible while still maintaining an acceptable level of expressive performance. We also limit ourselves to a low-cost solution for tactors: using vibrating motors. As a first step, we selected a well-studied task, tactile letter reading, to determine whether it is possible to achieve acceptable performance with an extremely reduced number of tactors. Specifically, we examined the ability to distinguish letters of the alphabet and numbers through vibrotactile stimuli provided by a 3-by-3 tactor array. We applied a "tracing mode" to display letters, which has been proven to yield very good accuracy in tactile letter reading. This method makes use of the spatio-temporal characteristics of sequential strokes in writing letters rather than showing a dot matrix pattern of the entire letter at once. We conducted an experiment to determine how accurately people can recognize the displayed letters. 


\section{Related Work}

Bach-y-Rita [1] and Collins [2] constructed a tactilevision substitution system (TVSS) with a 400 (20-by-20) pin array on the back. Because this system was developed to provide haptic substitution for television systems, initially letters were displayed as-is, i.e., vibrating pins were activated simultaneously to compose a dot array pattern of the letter as it is displayed on a visual display. This method was called "static mode." Loomis [3] used the same TVSS system and introduced a method to control the vibration stimuli such that the letters are "scanned" through a slit. He proposed two scanning techniques: a stationary letter scanned by a moving slit (moving-slit mode) and a moving letter scanned through a stationary slit (moving-letter mode). In both moving-slit and moving-letter mode, the performance of letter recognition was improved over that of static mode.

Saida et al. [4] constructed a small-sized (10-by-10) TVSS system and introduced a temporally sequential pattern of vibrotactile stimuli: tracing the letter stroke in the same order as hand writing. This method was called "tracing mode," and it achieved significant improvement in the accuracy of vibrotactile letter reading. They obtained 95\% correct letter recognition among the 46 letters of katakana (Japanese phonetic character set) in tracing mode, while the static or moving-letter mode yielded less than 50\% accuracy. Shimizu et al. [5] looked into the temporal parameters of tracing mode (duration of each stimulation point and inter-stroke interval) using a 7by- 9 dot pin array on the palm. They obtained more than $90 \%$ correct recognition among 46 katakana, with a certain condition of stimulation duration and inter-stroke interval.

More recently, researchers have developed a variety of vibrotactile displays; some were designed to expand the region of tactile interface to the whole body, and others introduced a tactile channel to human-computer interfaces for wearable computers.

Tan et al. discussed how to incorporate tactile displays to wearable computing systems [6] and combined input from pressure sensors mounted on the seat of an office chair with output in the form of vibrotactile units embedded in the back of the seat [7]. They also examined the effect of "sensory saltation" in order to display directional information by the spatio-temporal sequence of vibrating stimuli [8].

Kume et al. [9] introduced vibrotactile stimulation on the sole of the foot and developed a slipper-like interface. They put two tactors on each sole and made use of phantom sensations elicited by these tactors.

Yano et al. [10] developed a suit-type vibrotactile display with 12 tactors attached to the forehead (1), the palms (2), elbows (2), knees (2), thighs (2), abdomen (1), and back (one on the left side and one on the right). They examined the effectiveness of using this vibrotactile display for tasks that required the user to walk around a virtual corridor visually presented in a CAVE-like display.

Gunther et al. [11] also developed a suit-type vibrotactile display, to be used as a device for "tactual visualization" of music. They placed 12 high-frequency tactors on the shoulders, elbows, wrists, thighs, knees, and ankles, and one low-frequency tactor on the back.

Rupert [12] developed a system using a vest with tactors sewn into it to allow pilots to better judge the down-vector when performing aerial maneuvers that alter the pilot's vestibular system, which can cause fatal errors in judgment. He found that feedback to the torso could be effective in improving a pilot's spatial awareness.

Gemperle et al. [13] discussed the design strategy of wearable tactile displays and developed vest-style and harness-style displays that cover the user's torso.

Yang et al. [14] developed a belly-band-shaped vibrotactile display system, named "POS.T. Wear." They attached 60 tactors (5 layers; each layer has 12 tactors for horizontal orientation) to a tight-fitting T-shirt.

When constructing wearable vibrotactile display systems, the number and density of stimulation points are important design factors. Existing suit-type vibrotactile displays such as those by Yano et al. [9] and Gunther et al. [11] aim at the whole body, but the density of stimulating points in these systems was sparse (or discrete). On the other hand, the approaches by Tan et al. [6-8], Rupert [12], and Yang et al. [14] could be regarded as attempts to provide "analog" sensation at the locations of stimulation points, although the target regions only cover a limited area on the body surface. If we want to cover the whole body with tactors at considerable density, the total number of tactors would be huge, which might result in encumbering, easy-to-fail systems. Our interest lies in how to balance the number of tactors and the target region, i.e., how to reduce the number of tactors while maintaining acceptable performance by the vibrotactile display.

\section{Apparatus}

\subsection{Multi-channel Controller}

To control the tactor array (described later), we used the TactaBoard system [15]. This system incorporates the control of a large number of different types of feedback devices into a single, unified interface.

Using standard $2.5-\mathrm{mm}$ phono connectors, the system can be quickly reconfigured for use with various types and numbers of tactors. We have experimented with different deployment form-factors, such as an upper-body garment, stylus, glove, sleeve, and the office chair used in the current work. Each one of these form-factors used the same TactaBoard, only requiring the correct tactors to be 


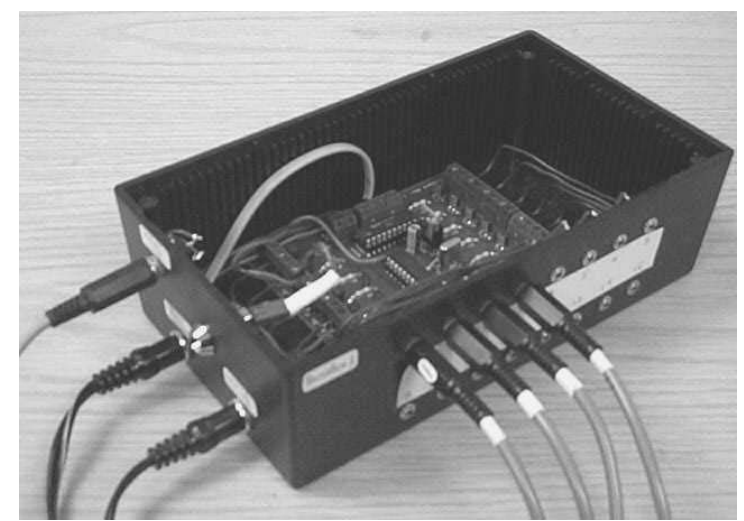

Figure 3. TactaBoard inside a box

plugged in. The power supply for the tactors is separate from the power for the circuit board, which allows output devices with fairly substantial power requirements to be supported.

In addition, the system can be run completely from battery power, and it uses a wireless connection to provide control from the host computer running the simulation software. The current version supports the independent control of 16 outputs on a single controller board using a standard serial port. Multiple boards can be controlled through the use of Bluetooth connectivity.

\subsection{Tactor Array}

A 3-by-3 array of tactors was affixed to an office chair, with a spacing of $6 \mathrm{~cm}$ between the centers of each pair of neighboring tactors.

The tactors in the lowest row were affixed such that they touched the back of the subject just above the belt line. The center column of tactors touched the subject along the spine. Care was taken to insure that subjects wore light clothing for the experimental session, and most wore dress shirts or T-shirts.

The tactors used in this setup were DC motors with an eccentric mass. They are manufactured by Tokyo Parts Industrial Co., Ltd. (Model No. FM23A) and have an operating voltage range of $0.8-1.6 \mathrm{~V}$ at $30 \mathrm{~mA}$. They have a standard speed of 5,000 RPM at $1.3 \mathrm{~V}$ and a vibration quantity of $1.0 \mathrm{G}$. Each of these disk-shaped tactors measures $18 \mathrm{~mm}$ in diameter and $3 \mathrm{~mm}$ in thickness. We operated the motors at $1.5 \mathrm{~V}$ for this experiment but modulated the signal sent to the tactors by using the TactaBoard.

The vibration aspect for tactors on the chair was slightly different from the standard specification. There was additional mechanical load due to the back of the chair and the sponge that the motors were attached to, and this load affected the vibration frequency of the tactor. The average vibration frequency was $69 \mathrm{~Hz}$ for attached

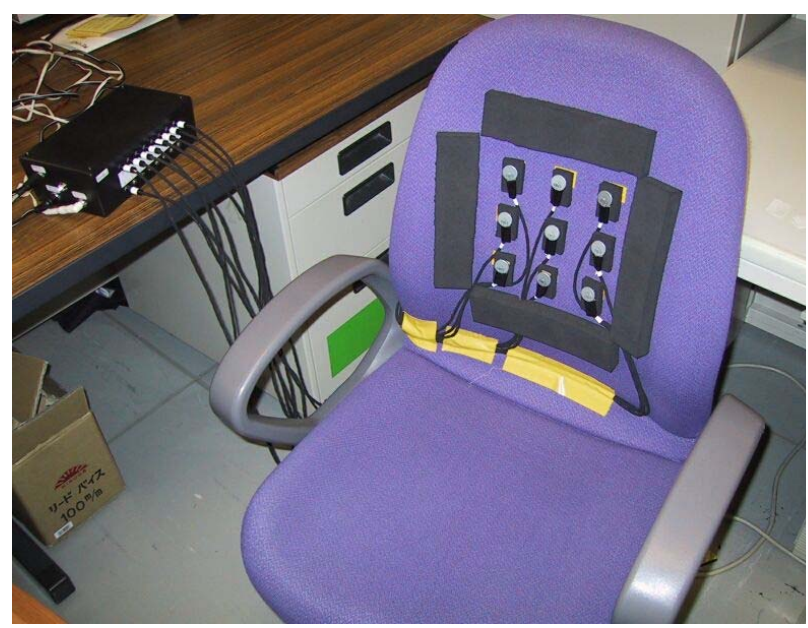

Figure 1. Office chair with 3-by-3 tactor array

motors and $85 \mathrm{~Hz}$ (almost equivalent to the specification) for free motors.

\section{Methods}

\subsection{Authoring stimulus sequences}

Unlike "static mode," "moving-slit mode," or "moving-letter mode," in which the locations of stimulation points are directly obtained from a visual font pattern, we had to provide a sequential pattern for each letter with a "tracing mode." As the tactor array size (3-

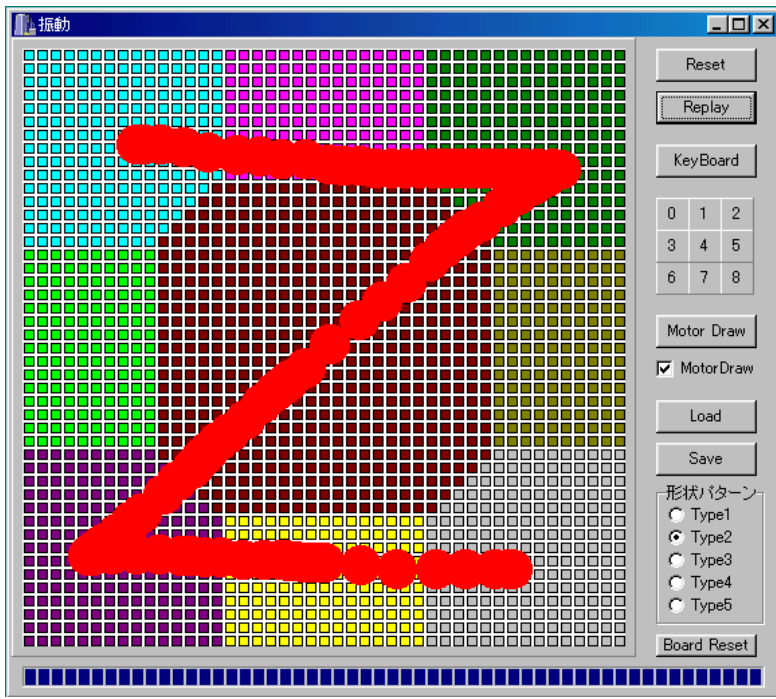

Figure 2. Utility to map mouse trajectory to stimulation points. The partitioning manner is not a simple grid; the central cell is in an octagon shape, so the diagonal strokes only cross the three diagonal cells. 


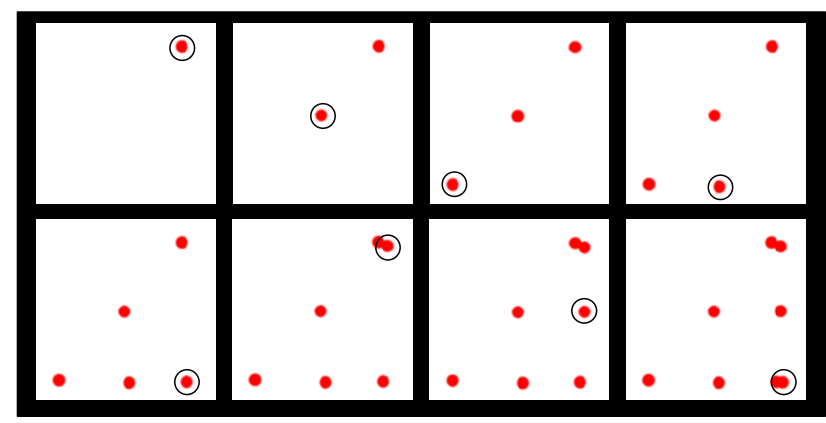

(a) Number "4"

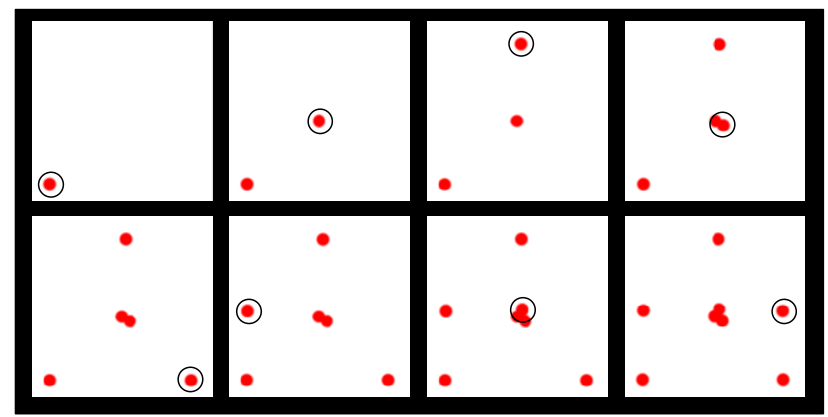

(b) Letter "A"

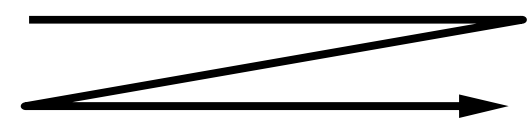

Figure 4. Examples of sequential patterns to display letters and numbers: (a) "4" and (b) "A." The sequence goes from left to right in the upper row and then left to right in the lower row. Each dot represents a stimulation point within the 3-by-3 array. Overlapped dots indicate the same stimulation point, and the slight difference in position is just to show these points are stimulated more than one time. In each letter, there is an inter-stroke interval between the fifth and sixth stimulation points.

by-3) of our system is not sufficient to represent all of the alphanumeric characters by simultaneously activating the tactors composing the dot matrix pattern for each letter, this was not a simple tracing procedure.

Nevertheless, we can still feel the direction and rough position of the stroke with a 3-by-3 tactor array. We decided to make use of the characteristic strokes composing each letter rather than exactly decomposing the pen trajectory into positions of the stimulation points. To do this, we made an interface program to map the hand-written stroke into a sequence of stimulation points. Figure 3 shows the mapping interface used to generate the sequence of stimulation points based on hand-written letters. With this utility, we can record the time and position of the mouse cursor and playback the recorded sequences.

After generating the sequence of stimulation points for each letter, we extracted the information on the order of position and stroke continuity, discarding the duration of each stimulation point. This was done to avoid variation in pen speed in handwriting the letter, thus providing a uniform sequence of stimuli. The final results of the authoring are shown in Figure 4. We generated stimulation sequences for numbers and capital alphabet letters (10 digits plus 26 letters). The sequences for "O" and " 0 " (zero), and " $Z$ " and " 2 " are identical, so there were 34 patterns in total.

\subsection{Temporal Aspects}

To display letters using "tracing mode", temporal issues are very important [5], since the successive point of stimulation within a single stroke of the letter should be perceived as a continuous stroke while the inter-stroke interval should be perceived as "pen-up."

At first we tried stimulation sequences with approximately $200 \mathrm{~ms}$ of duration for one point, based on the results of previous research $[16,8]$, but the subjective impression was not good from our preliminary observation. It was often difficult to identify which tactor was being activated. This phenomenon is most likely caused by the difference in tactor devices between our experimental setup and that of the previous research. We used vibrating motors as tactors because vibrating motors are low-cost, have low power consumption, and do not require complicated driving circuits. However, their temporal response at onset and breaking instant is rather dull. It takes $100-200 \mathrm{~ms}$ for them to vibrate at full amplitude, and even more time to decay after the control voltage is removed.

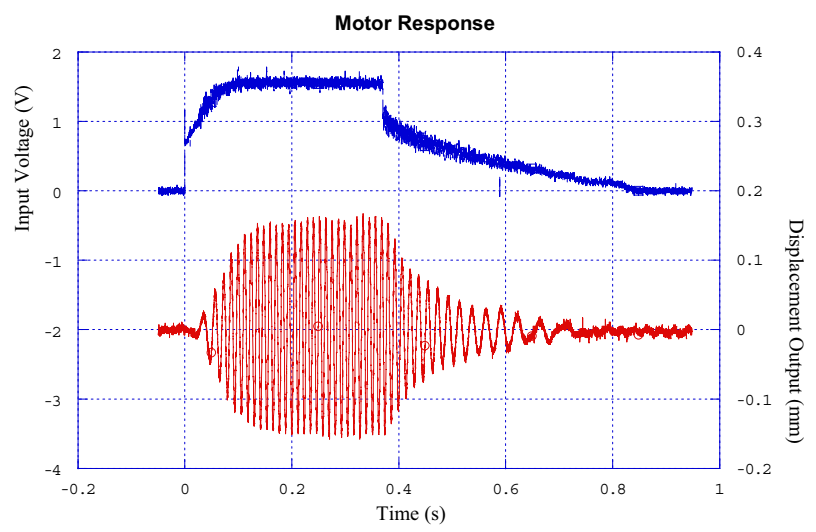

Figure 5. Response of a vibrating motor to stepwise voltage input. 
We measured the motor response using a laser range finder system. We used models LK-2000 (main unit) and LK-030 (head unit) from Keyence Corporation, and the output of the sensor unit was fed to a digital oscilloscope (Tektronix 3014B) together with the voltage signal driving the vibrating motor. Figure 5 shows the response of the vibrating motor (lower plot) to the step-wise control voltage input (upper plot).

We conducted a preliminary experiment to find acceptable (though not optimized) stimulus duration and inter-stimulus onset interval (ISOI), often called stimulus onset asynchrony (SOA). We decided to use a duration of $500 \mathrm{~ms}$ for each stimulation point and the same length of ISOI, i.e., simply switching the tactors from one to another. Here, we avoided the use of an ISOI shorter than stimulus duration because simultaneous activation of multiple tactors caused an unnatural beat in our system. The inter-stroke interval was also set to $500 \mathrm{~ms}$. As the result of stimulation pattern authoring, the total display duration for each character ranged from 1.5 seconds (number "1") to 7.5 seconds (letter "B").

\subsection{Procedure}

At the beginning of the experiment, the subject was asked to confirm the feeling of vibration stimuli and the location of tactors by activating each of nine tactors manually. Then, the experimental session was started. Each session was composed of 100 trials, which are categorized in three conditions: 38 trials with randomlyselected numeric characters, 52 trials with 2 sets of alphabet characters in random order, and 10 trials with randomly-selected alphanumeric (mixed) characters.

\subsection{Subjects}

The subjects were 10 people ( 8 males and 2 females), including researchers and intern students at ATR, ranging in age from 22 to 39 . They volunteered for participation in a 10-20 minute experimental session. All subjects were familiar with the alphabet, though they were not native English speakers.

\section{Results and Discussion}

\subsection{Results}

The subjects could answer the recognized letter or number just after the sequence for each letter or number was completed, usually within 1 second. For some trials, however, they took more than several seconds when they were not confident about the recognition result.

The ratio of correct letter recognition is shown in Figure 6 . The total ratio of correct letter recognition

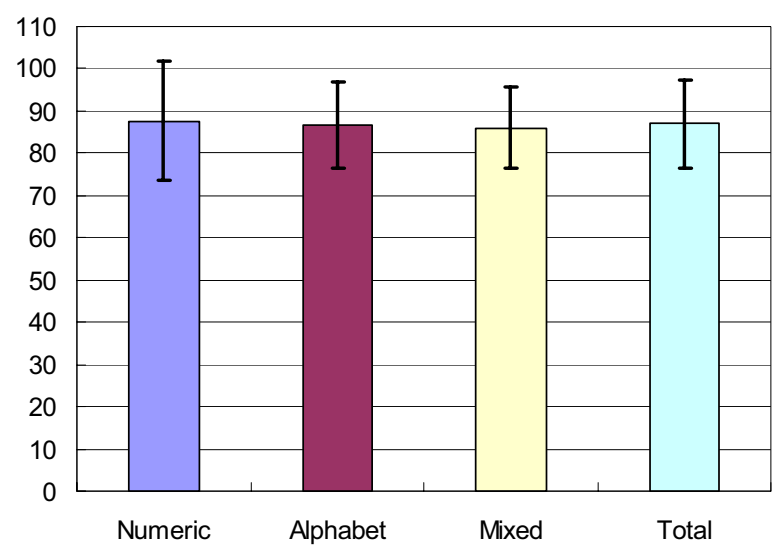

Figure 6. Accuracy of letter recognition.

(accuracy) for all subjects was $87 \%$. The values of accuracy were $87.6 \%$ for the set of numeric characters, $86.7 \%$ for the set of alphabet characters, and $86 \%$ for the set of alphanumeric (mixed) characters. The standard deviations among the 10 subjects for all trials, numeric, alphabetic, and mixed character sets were $10.4 \%, 14.1 \%$, $10.1 \%$, and $9.7 \%$, respectively. There were no statistical significances between the numeric and alphabetic character sets $(p>0.1)$.

Among the numeric letters, the number " 1 " yielded the best accuracy (100\%), followed by " 8 ", "5", "6", "9", "4", "7", " 0 ", and " 2 ", with " 3 " being the worst $(77.8 \%)$. For alphabet letters, "E", "O", "Q", and "T" yielded the best accuracy $(100 \%)$, and "S" was the worst $(62.1 \%)$.

\subsection{Discussion}

In terms of the accuracy in vibrotactile letter reading, the results of our experiment are comparable to previous research using larger arrays of tactors; for example, 95\% with a 10-by-10 tactor array on the abdomen by Saida et al. [4] and $80-95 \%$ with a 7-by-9 array on the palm by Shimizu et al. [5] In these works, they used 46 katakana characters, so our results (34 alphanumeric letters) could not be directly compared. However, our result of $87 \%$ was much better than the result with a 20-by-20 array for alphabet recognition by Loomis [3], where average recognition accuracy was $51 \%$ ("moving-letter mode") or worse ("static mode"). This supports the effectiveness of using "tracing mode."

The results show that we can provide a greater variety of expression if we make use of the information of the stroke, i.e., motion-related information. For example, number " 8 " and letter " $\mathrm{B}$ " generated the same dot matrix pattern, but we can distinguish these two characters by the order of activated tactors. In the previous research [3, 4], the use of "tracing mode" was significant for better 
performance, but in our case there is a difference in the number of expressible characters, in principle.

In our experiment, there was no significant difference in accuracy between selecting characters from 10 digits and from 26 alphabet letters. This implies that the limit of the number of expressible characters exceeds 26. Although the number of trials is much smaller than the numeric and alphabetic cases, mixed (alphanumeric) case yielded almost the same accuracy, so the number of expressible characters might exceed 34. Now let us consider this number in principle. Assuming three horizontal strokes, three vertical strokes, four diagonal strokes, and two (clockwise and counter-clockwise) round strokes can be distinguished with our system, giving 12 in total, and that the stroke interval can definitely be detected, then it's possible to distinguish, in principle, 12 single-stroke characters, 144 two-stroke characters, and 1,728 three-stroke characters. This number is sufficient to cover all alphanumeric characters.

Hence, one key factor affecting the recognition accuracy is how accurately the subject can detect the characteristics of each stroke. Interestingly, the accuracy of letter reading was quite close to the correct identification rate of tactor position $(84 \%)$ in our previous experiment [17]. This implies that further improving the accuracy of letter reading might be achieved by raising the performance of identifying the tactor's position.

Familiarity with the displayed character set may also affect accuracy. For example, the result of accuracy might be different if katakana is displayed to a subject who is not familiar with Japanese characters. This should be taken into account if we want to design original or modified character sets (such as Graffiti for Palm devices) to make full use of stroke characteristics. One merit of designing such a modified or custom character set would be to keep some distance metric of similarity between characters. For example, subjects could not distinguish " 2 " and "Z" because our system does not have the ability to express the difference between a round curve and an angle. It might be possible to distinguish these two by making some modification or imposing some rules on the character set. In historical research, Kikuchi et al. [19] measured the misrecognition among alphabets with their TVSS system ("static mode") to compose a letterconfusion matrix and analyzed the proximity of every combination of two letters. They found that at least three features of letters play important roles in tactile letter recognition. It might be useful to do similar analysis for "tracing mode."

One major problem with our method was that it took a relatively long time to show a letter (several seconds per letter). In order to reduce the stimulating time per letter, the use of apparent motion $[16,18]$ is proposed. With this method, a single stroke is expressed by two (start and end) points, driven so that the tactile apparatus motion is perceived. According to Shimizu et al. [20], 50\% time reduction was achieved with their system. Here, however, we face an undesirable characteristic in our system: the dull temporal response of vibrating motors, which is different from the previous research. Nevertheless, we believe we can find the optimal temporal pattern to invoke apparent motions by using vibrating motors in the future. Kirman [21] reported that the optimal values of ISOI are decreased when the number of sequentially activated tactors is increased from two to four, which can be regarded as positive evidence for the ability to achieve efficient information display by low-resolution vibrotactile display systems.

\section{Conclusions and Future Work}

We examined the possibility of showing alphanumeric letters by using only a 3-by-3 tactor array, and subjects could successfully distinguish most alphanumeric letters by making use of characteristic strokes. This result was better than we expected, considering that it's only barely possible to represent alphanumeric letters statically with a 3-by-3 dot matrix. Our findings also support the design strategy of wearable vibrotactile displays by Tan et al. [6], in that the use of directional information is effective.

Further studies will include finding optimal timing to drive tactors and finding a general rule for optimal authoring of stimulus sequences. We also plan to apply a similar strategy to other parts of the body surface by constructing wearable vibrotactile display systems.

\section{Acknowledgement}

This research was supported in part by the Telecommunications Advancement Organization of Japan.

\section{References}

[1] P. Bach-y-Rita, Brain mechanisms in sensory substitution, Academic Press, New York, 1972.

[2] C. C. Collins, "Tactile television-Mechanical and electrical image projection," IEEE Transactions on Man-Machine System, MMS-11, 1970, pp. 65-71.

[3] J. Loomis, "Tactile letter recognition under different modes of stimulus presentation," Perception \& Psychophysics, Vol. 16, No. 2, 1974, pp. 401-408.

[4] S. Saida, Y. Shimizu, and T. Wake, "Construction of Small TVSS and Optimal Mode of Stimulus Presentation," 4th Symposium on Sensory Substitution, Ibaraki, Japan, 1978, pp. 68-73 (in Japanese). 
[5] Y. Shimizu, "Temporal Effect on Tactile Letter Recognition by a Tracing Mode," Perceptual and Motor Skills, 55, 1982, pp. 343-349.

[6] H. Tan and A. Pentland, "Tactile Displays for Wearable Computing," First International Symposium on Wearable Computers, 1997, pp. 8489.

[7] H. Tan, I. Lu, and A. Pentland, "The Chair as a Novel Haptic User Interface," Proc. of the Workshop on Perceptual User Interfaces, 1997, pp. 56-57.

[8] H. Tan, A. Lim, and R. Traylor: "A Psychophysical Study of Sensory Saltation with an Open Response Paradigm," Proc. of 9th International Symposium on Haptic Interfaces for Virtual Environment and Teleoperator Systems, ASME Dynamic Systems and Control Division, Vol. 69-2, 2000, pp. 1109-1115.

[9] Y. Kume, A. Shirai, M. Tsuda, and T. Hatada, "Information transmission through soles by vibrotactile stimulation," Trans. of the Virtual Reality Society of Japan. Vol. 3, No. 3, 1998, pp. 83-88 (in Japanese).

[10] H. Yano, T. Ogi, and M. Hirose, "Development of Haptic Suit for Whole Human Body Using Vibrators," Trans. of the Virtual Reality Society of Japan, Vol. 3, No. 3, 1998, pp. 141-148 (in Japanese).

[11] E. Gunther, G. Davenport and S. O'Modhrain, "Cutaneous Grooves: Composing for the Sense of Touch," Proc. 2002 Conference on New Interfaces for Musical Expression (NIME-02), 2002, pp. 37-42.

[12] A. H. Rupert, "An Instrumentation Solution for Reducing Spatial Disorientation Mishaps," IEEE Engineering in Medicine and Biology Magazine, 19(2), 2000, pp. 71-80.
[13] F. Gemperle, N. Ota, and D. Siewiorek, "Design of a Wearable Tactile Display," Proc. of IEEE 5th International Symposium on Wearable Computers, 2001, pp. 5-12.

[14] U. Yang, Y. Jan, and G. J. Kim, "Designing a VibroTactile Wear for Close Range Interaction for VRbased Motion Training," Proc. 12th International Conference on Artificial Reality and Telexistence (ICAT2002), 2002, pp. 4-9.

[15] R. W. Lindeman and J. R. Cutler, "Controller Design for a Wearable, Near-Field Haptic Display," Proc. of 11th International Symposium on Haptic Interfaces for Virtual Environment and Teleoperator Systems, 2003, pp. 397-403.

[16] C. E. Sherrick and R. Rogers, "Apparent haptic movement," Perception \& Psychophysics, Vol. 1, 1966, pp. 175-180.

[17] R. W. Lindeman and Y. Yanagida, "Empirical Studies for Effective Near-Field Haptics in virtual Environments," Proc. of IEEE Virtual Reality 2003, pp. 287-288.

[18] J. H. Kirman, "Tactile apparent movement: The effects of interstimulus onset interval and stimulus duration," Perception \& Psychophysics, Vol. 15, No. 1, 1974, pp. 1-6.

[19] T. Kikuchi, Y. Yamashita, K. Sagawa, and T. Wake, "An analysis of tactile letter confusions," Perception \& Psychophysics, 26(4), 1979, pp. 295-301.

[20] Y. Shimizu, "Temporal Factors for Vibrotactile Letter Reading," Perceptual and Motor Skills, 80, 1995, pp. 1069-1070.

[21] J. H. Kirman, "The effect of number of stimulators on the optimal interstimulus onset interval in tactile apparent movement," Perception \& Psychophysics, Vol. 17, No. 3, 1975, pp. 263-267. 